\title{
Correction to: Illite crystallinity index from the Mesoproterozoic sedimentary cover of the Kaladgi basin, southwestern India: Implications on crustal depths of subsidence and deformation
}

\author{
Mrinal Kanti Mukherjee ${ }^{1, *}$, Kunal ModaK ${ }^{1}$ and Jiten Ghosh ${ }^{2}$ \\ ${ }^{1}$ Department of Applied Geology, Indian Institute of Technology (Indian School of Mines), \\ Dhanbad 826004, Jharkhand, India. \\ ${ }^{2}$ Advanced Mechanical and Material Characterization Division, CSIR-Central Glass and Ceramics Research \\ Institute, Jadavpur, Kolkata 700032, India. \\ *Corresponding author.e-mail: mrinal_km67@yahoo.co.in
}

published online 4 May 2019

Correction to: J. Earth Syst. Sci. (2019) 128:101; https://doi.org/10.1007/s12040-019-1124-7

In the original version of this article, figure 2 and table 3 were incorrectly represented. The corrected figure 2 and table 3 are given below.

The original article has been corrected. 


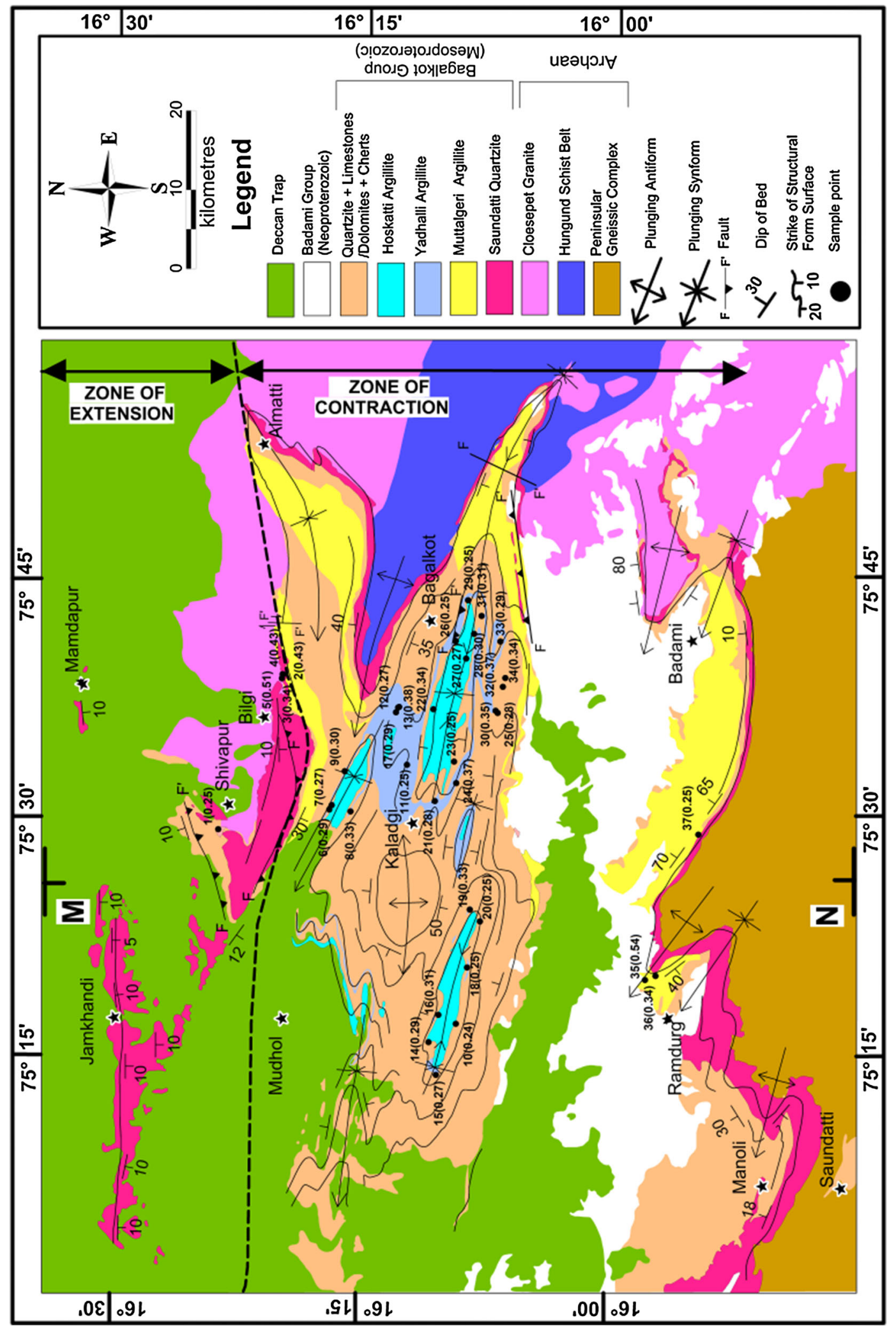

嵌

$\rightarrow$.

ॠ

00

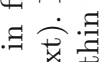

娄

蔗芯

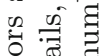

芯 :

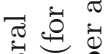

궁 의

1.

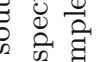

T证

का एँ

घ :

점

.

菏

is

.

जี

늄

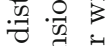

\%

$\exists$.

శี

สี

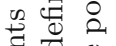

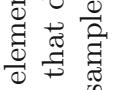

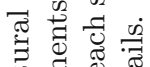

进范

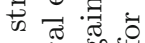

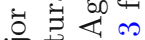

范苛

$\Leftrightarrow$

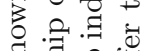

का जी

敢.

它氙范

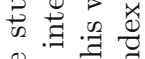

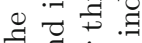

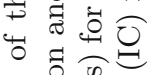

ब्वै:

శె

.0.

응

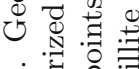

궁

芯芯 范 
Table 3. Measured FWHM, calibrated FWHM (IC CIS ) using CIS of Warr and Rice (1994) (table 2 of this work) and Kubler equivalent FWHM (IC Kubler) of the 10- $\AA$ basal reflection of the illite-muscovite in XRD analysis of the argillites Kaladgi basin.

\begin{tabular}{|c|c|c|c|c|c|c|c|}
\hline $\begin{array}{l}\text { Sample } \\
\text { no. }\end{array}$ & Sample code & Stratigraphic identity & Northing & Easting & $\begin{array}{l}\text { FWHM } \\
\text { (measured) }\end{array}$ & $I C_{\mathrm{CIS}}$ & $I C_{\text {Kubler }}$ \\
\hline $1^{\#}$ & $22 \mathrm{D} / 7(2014)$ & Saundatti Quartzite & 16.3936111 & 75.48639 & 0.1973667 & 0.28 & 0.25 \\
\hline $2^{*}$ & 13JN/7 (2011) & Muttalgeri Argillite & 16.3286111 & 75.64917 & 0.307 & 0.51 & 0.43 \\
\hline $3^{*}$ & 9OC/1 (2013) & Saundatti Quartzite & 16.3294444 & 75.64472 & 0.24 & 0.40 & 0.34 \\
\hline $4^{*}$ & 13JN/5 (2011) & Muttalgeri Argillite & 16.3286111 & 75.64889 & 0.3053 & 0.51 & 0.43 \\
\hline $5^{*}$ & 13JN/6 (2011) & Muttalgeri Argillite & 16.3283333 & 75.64861 & 0.36 & 0.60 & 0.51 \\
\hline $6^{*}$ & $7 \mathrm{OC} / 8(2013)$ & Yadhalli Argillite & 16.2816667 & 75.50694 & 0.201 & 0.33 & 0.29 \\
\hline $7^{*}$ & $7 \mathrm{OC} / 4(2013)$ & Hoskatti Argillite & 16.2791667 & 75.51167 & 0.1877 & 0.31 & 0.27 \\
\hline $8^{*}$ & 15D/4 (2013) & Yadhalli Argillite & 16.2602778 & 75.505 & 0.2301 & 0.38 & 0.33 \\
\hline $9^{*}$ & 14D/1 (2013) & Yadhalli Argillite & 16.2661111 & 75.54722 & 0.2093 & 0.35 & 0.30 \\
\hline $10^{*}$ & $31 \mathrm{~J} / 4(2014)$ & Hoskatti Argillite & 16.1541667 & 75.28194 & 0.1605 & 0.27 & 0.24 \\
\hline $11^{\#}$ & 14J/4 (2015) & Yadhalli Argillite & 16.2033333 & 75.55389 & 0.1985667 & 0.29 & 0.25 \\
\hline $12^{*}$ & $3 \mathrm{~J} / 2(2013)$ & Yadhalli Argillite & 16.2122222 & 75.61417 & 0.1878 & 0.31 & 0.27 \\
\hline $13^{*}$ & $4 \mathrm{M} / 5(2011)$ & Yadhalli Argillite & 16.2111111 & 75.61472 & 0.269 & 0.45 & 0.38 \\
\hline $14^{*}$ & $21 \mathrm{D} / 7(2013)$ & Hoskatti Argillite & 16.1816667 & 75.26278 & 0.2001 & 0.33 & 0.29 \\
\hline $15^{*}$ & $23 \mathrm{D} / 3(2013)$ & Yadhalli Argillite & 16.1741667 & 75.22833 & 0.1847 & 0.31 & 0.27 \\
\hline $16^{*}$ & $26 \mathrm{~J} / 9$ (2014) & Hoskatti Argillite & 16.1716667 & 75.29139 & 0.2167 & 0.36 & 0.31 \\
\hline $17^{*}$ & 3J/4 (2013) & Yadhalli Argillite & 16.2144444 & 75.60917 & 0.2042 & 0.34 & 0.29 \\
\hline $18^{*}$ & 17D/4 (2013) & Yadhalli Argillite & 16.1427778 & 75.34111 & 0.1728 & 0.29 & 0.25 \\
\hline $19^{*}$ & $6 \mathrm{OC} / 7(2013)$ & Yadhalli Argillite & 16.14 & 75.40194 & 0.2275 & 0.38 & 0.33 \\
\hline $20^{*}$ & $20 \mathrm{D} / 3(2013)$ & Yadhalli Argillite & 16.1297222 & 75.38944 & 0.1713 & 0.28 & 0.25 \\
\hline $21^{*}$ & 19JN/5 (2011) & Yadhalli Argillite & 16.1752778 & 75.51583 & 0.1909 & 0.32 & 0.28 \\
\hline $22^{*}$ & 3J/1 (2013) & Hoskatti Argillite & 16.1766667 & 75.61222 & 0.24 & 0.40 & 0.34 \\
\hline $23^{*}$ & 17JN/3 (2012) & Yadhalli Argillite & 16.1558333 & 75.55722 & 0.1701 & 0.28 & 0.25 \\
\hline $24^{*}$ & $5 \mathrm{~J} / 6(2013)$ & Yadhalli Argillite & 16.1536111 & 75.53472 & 0.259 & 0.43 & 0.37 \\
\hline $25^{*}$ & $5 \mathrm{~J} / 4 \mathrm{~B}(2013)$ & Yadhalli Argillite & 16.1119444 & 75.60833 & 0.1953 & 0.32 & 0.28 \\
\hline $26^{*}$ & $3 \mathrm{M} / 7$ (2011) & Yadhalli Argillite & 16.1541667 & 75.68389 & 0.1678 & 0.28 & 0.25 \\
\hline $27^{*}$ & $5 \mathrm{M} / 8(2011)$ & Hoskatti Argillite & 16.1436111 & 75.66556 & 0.1894 & 0.31 & 0.27 \\
\hline $28^{*}$ & $5 \mathrm{M} / 7(2011)$ & Hoskatti Argillite & 16.1355556 & 75.69139 & 0.2118 & 0.35 & 0.30 \\
\hline $29 *$ & $2 \mathrm{~J} / 3$ (2011) & Hoskatti Argillite & 16.1416667 & 75.72667 & 0.1761 & 0.29 & 0.25 \\
\hline $30 *$ & $5 \mathrm{~J} / 3(2013)$ & Yadhalli Argillite & 16.1138889 & 75.61056 & 0.2416 & 0.40 & 0.35 \\
\hline $31^{*}$ & $28 \mathrm{~F} / 5(2011)$ & Hoskatti Argillite & 16.1280556 & 75.71028 & 0.2161 & 0.36 & 0.31 \\
\hline $32^{*}$ & $5 \mathrm{OC} / 6$ & Yadhalli Argillite & 16.1066667 & 75.63583 & 0.2602 & 0.43 & 0.37 \\
\hline $33^{*}$ & 13JN/3 (2012) & Hoskatti Argillite & 16.1091667 & 75.68333 & 0.2042 & 0.34 & 0.29 \\
\hline $34^{*}$ & $15 \mathrm{JN} / 2$ & Yadhalli Argillite & 16.1041667 & 75.645 & 0.24 & 0.40 & 0.34 \\
\hline $35^{\#}$ & 23D/1 (2014) & Muttalgeri Argillite & 15.9658333 & 75.3292 & 0.507933 & 0.64 & 0.54 \\
\hline $36^{\#}$ & $23 \mathrm{D} / 3(2014)$ & Muttalgeri Argillite & 15.9525 & 75.34194 & 0.2888 & 0.39 & 0.34 \\
\hline $37^{\#}$ & $13 \mathrm{JN} / 3 \mathrm{~A}(2015)$ & Muttalgeri Argillite & 15.9094444 & 75.48056 & 0.20303 & 0.29 & 0.25 \\
\hline
\end{tabular}

\#Batch-1 samples: calibration equation is $I C_{\mathrm{CIS}}=1.16 \times I C_{\text {measured }}+0.0559\left(R^{2}=0.984\right)$.

*Batch-2 samples: calibration equation is $I C_{\mathrm{CIS}}=1.664 \times I C_{\text {measured }}-0.0001\left(R^{2}=0.951\right)$. 